\title{
On-Line Adaptive Filtering of Web Pages
}

\author{
Richard Nock ${ }^{1}$ and Babak Esfandiari ${ }^{2}$ \\ 1 GrimaAg, Université Antilles-Guyane, Schoelcher, France \\ rnock@martinique.univ-ag.fr \\ 2 Dept of Systems and Computer Engineering, \\ Carleton University, Ottawa, Canada \\ babak@sce.carleton.ca
}

\begin{abstract}
We present a browser extension to dynamically learn to filter unwanted Uniform Resource Locators (such as advertisements or flashy images) based on minimal user feedback. Our extension builds upon one of the top ten of Mozilla firefox plug-ins which filters URLs without learning capabilities. We apply a weighted majority-type learning algorithm working on regular expressions. Experimental results confirm that the accuracy of the predictions converges quickly to very high levels, with other key parameters: recall, specificity and precision.
\end{abstract}

\section{Introduction}

Many attempts have been made to make Web browsing more pleasant by allowing the user to remove big pictures and unwanted animations that interfere with reading. Some browsers such as Netscape or Mozilla allow the user to collapse such pictures or even create blacklists of internet domains that supply them.

But the most sophisticated approach so far has been proposed by the developers of AdBlock. AdBlock [1] is, according to "Mozdev update" data 6], in the top ten of the most popular extension to the Mozilla Firefox web browser [5], with about 100000 downloads. To use AdBlock, the user has to come up with a collection of regular expressions that describe the URL patterns of images that they want to see filtered. As a result, whenever the browser is pointed to an item whose URL is matched by a regular expression, it is simply ignored, which not only "cleans up" the web page, but also makes page downloading faster.

\footnotetext{
$/[\wedge a-z \backslash d=+\%](\backslash w * \backslash d+x \backslash d) ? \backslash d *($ show)? (\w\{3,\}\%20|alligator $\mid$ avs $\mid$ barter $\mid$ blog $\mid$ box $\mid$ central $|d ? h t m l| i ?$ frame $\mid$ front $\mid$ fuse $\mid$ get $\mid$ house $\mid$ inline $\mid$

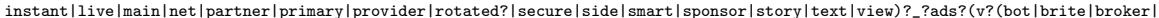

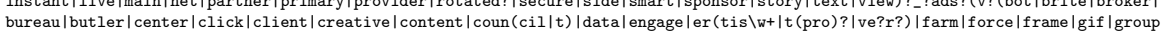

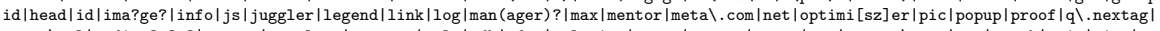
quest $\backslash . n l \mid$ redire?c?t? |remote|revolver|rotator|sale|sdk|sfac|solution|sonar|source|space|srv|stat.*1.asp|sys|track|trix|view|ty

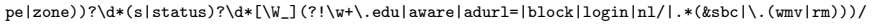

Fig. 1. Example of a long regular expression found on AdBlock's forum

However, as often discussed in the AdBlock online forum, coming up with regular expressions is a difficult task, especially for the non-computer savvy. Writing and mastering them accurately requires extensive readings [2], and those published on-line can be especially hard to read and understand. Figure 1 presents 
the example of a regular expression posted on AdBlock's forum. Most of the regular expressions posted are smaller than this one, but some of them appear to be much more complicated to understand. Thus, the user faces the risk of obtaining unwanted browsing/blocking behaviors, sometimes without really knowing how to correct them. The problem cannot be solved from a global standpoint, as it would be impossible to come up with a general set of filters that would satisfy every user. Finally, as the advertisement suppliers and browsing habits change, so should the set of regular expressions that are needed. The behavior of AdBlock is too static to be suited to these dynamic interactions, but moving to a dynamic interaction between the user and the filter is everything but trivial. The comfort the extension brings to the user has to be greater than its eventual drawbacks, and the complexity of the algorithm is clearly such a potential drawback.

To address this problem, we propose a fast machine learning approach that would create filters based on minimal interaction with the user. The user is not required to know how to create regular expressions; all that is required is for the user to click on URLs (e.g. images) that he/she wants to see blocked. Conversely, from time to time the user will need to unblock URLs that shouldn't have been blocked by the adaptive filter. Based on this simple feedback, our proposed method, an adaptation of the well known Weighted Majority algorithm [4, builds a set of "experts" (simple regular expressions on URLs) that vote on whether a given URL should be blocked or not.

In the following Section, we review some works related to our topic. Then, the next Section is devoted to a formal presentation of the algorithm. After a Section presenting the browser extension, a Section presents and discusses experimental results. A last Section concludes and presents relevant issues on the topic.

\section{Related Work}

Our approach is inspired by the concept of Interface Agents [3. An interface agent is a piece of software that assists a user of a complex system by observing his/her behavior and detecting patterns that it could reproduce in order to automate tedious tasks. Typically such programs use some kind of incremental machine learning algorithm to build the knowledge base. [3] devised interface agents that used $k$-nearest neighbor to classify mail and even share the filters with other agents. In a previous work, we used an adaptation of the Version Spaces algorithm to automate simple network management tasks [7].

But the closest work is perhaps the use of Bayesian filtering for detecting email spam [8], which is now a standard feature in mainstream email programs. Bayesian methods for filtering emails have the advantage of being conceptually simple, and a great body of previous work has made them tailored to common text classification tasks.

In our case, however, the setting makes them a priori not the best classification tool suited for web browsing. Classification is indeed made on-line. This is a crucial remark because the frequency of browsing through URLs is much higher than that of email receipt for the average user. This makes it necessary to have 
an ultra-fast classification tool with easy updates on the classifier, to filter the URLs as they come. In the case of email spam detection, it is already necessary to have efficient feature selection algorithms to reduce the vector space to a small set prior to using Bayesian methods [8. Making the additional heavy-weight online updates for URL filtering, such as the computation of the probability table for each feature, would rapidly slow down the browser and make its use very uncomfortable. Furthermore, Bayesian methods rely on independence assumptions (at best partially relaxed) on the features to make the classification sound 8. This is clearly not a desirable assumption for URL classification, since it partially omits token positions and contexts in an URL.

\section{Theoretical Setting}

Very informally, the algorithm can be reduced to the following infinite loop: get an example, update a set of experts and update the weights of each expert. Any time during the algorithm, a prediction is possible on an observation by using a weighted majority over the current set of experts.

More formally, each observation belongs to a set $X$, which contains all possible observations. Each observation is an URL (Uniform Resource Locator). From the user's standpoint, $X$ can be partitioned into two subsets. The first one contains the URLs he would like to block, i.e. refrain from loading. The other one contains all the other URLs, i.e. those he wishes to leave unblocked. To each URL can thus be associated a status which we call a class or label (block/unblock), and our objective is to predict the class of each URL as accurately as possible with respect to the user, given that any two different users may probably correspond to different partitions of $X$. Our algorithm builds therefore a decision function (or classifier) from $X$ onto $\{-1,+1\}$, with " +1 " denoting the class of the URLs to be blocked (also called the positive class), and " -1 " the class of the URLs to leave unblocked (the negative class).

We denote a couple (observation, class) obtained from the user as an example. We let $\left(x_{1}, y_{1}\right),\left(x_{2}, y_{2}\right), \ldots$ denote the stream of examples observed from the user, and $\left(x_{t}, y_{t}\right)$ is thus the $t^{t h}$ example of the stream. We build a set of experts $\mathbf{E}$ which is growing with time; to keep notations clear, we do not use the time subscript on $\mathbf{E}$ : it should be clear from context which set of experts we use. Each expert of $\mathbf{E}$ is a couple (hypothesis, weight). An hypothesis is a function $h: X \rightarrow\{-1,0,+1\}$ which is allowed to abstain (this is the output " 0 "). More precisely, each hypothesis' output is either $\{-1,0\}$ or $\{0,+1\}$, which means that the corresponding expert is authorized to say "I don't know", thus delegating the decision on the class of an observation to the other experts. The weight associated to hypothesis $h$ is denoted $w_{t}(h) \in \mathbb{R}^{+}$. It is a function of $t$ since it is updated each time an example is received. At the very beginning of the algorithm, prior to seeing the first example, we initialize the following set of parameters:

$-\beta \in(0,1)$ is a learning constant chosen by the user,

$-\mathbf{E} \leftarrow \emptyset$ is the initial set of experts,

$-t \leftarrow 1$ is the "time stamp" labeling the examples received. 
Algorithm 1 below displays more formally what happens when example $\left(x_{t}, y_{t}\right)$ is received.

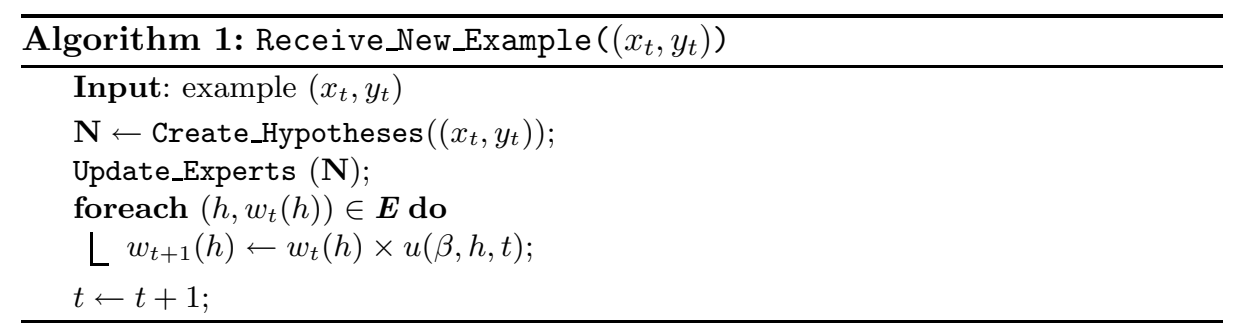

There are two possible choices for function $u(\beta, h, t)$ :

$$
u(\beta, h, t)=\frac{1+y_{t} h\left(x_{t}\right)}{2 \beta}+\frac{\left(1-y_{t} h\left(x_{t}\right)\right) \beta}{2} .
$$

There are two procedures in Algorithm[1. Create_Hypotheses(.) takes an example as input, and outputs a set of hypotheses (i.e. regular expressions). Since the theory underlying the algorithm does not depend on this procedure, we postpone the details and its implementation to the experimental section.

Update_Experts (.) takes as input a set of hypotheses, and creates a set of experts which is used to grow E. In other words, it initializes the weights of the hypotheses. Details are given in Algorithm 2 (here, "0" denotes the function which is zero everywhere in $\mathbb{R}$ ).

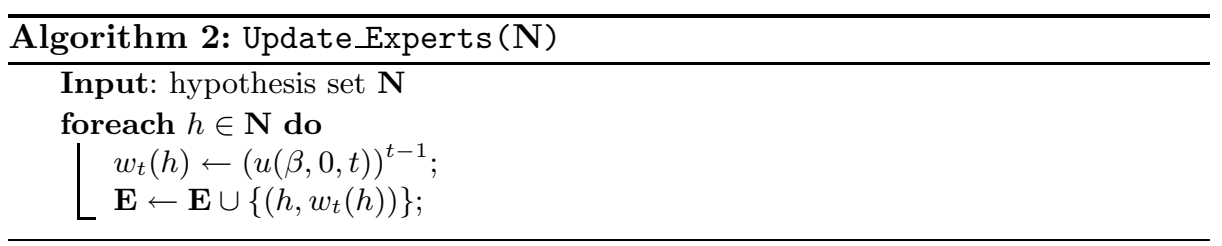

Weight initialization for new experts makes it possible to consider from the theoretical standpoint that each of them was created at the beginning of the algorithm, as everything is like if it were abstaining until "really" put into $\mathbf{E}$. There remains to give the way $\mathbf{E}$ is used to classify an observation $x \in X$. Just prior to receiving example $t+1$, the decision made out of $\mathbf{E}, H_{\mathbf{E}, t}$, relies on an ordinary majority vote: $\forall x \in X, H_{\mathbf{E}, t}(x)=\operatorname{sign}\left(\sum_{\left(h, w_{t}(h)\right) \in \mathbf{E}} w_{t}(h) \times h(x)\right)$.

\section{Design of the Browser Extension}

The Mozilla Firefox Web browser [5] is an open source product with an architecture specifically designed for allowing 3rd party extensions. This makes it 
possible to easily modify the browser behavior by overriding or augmenting the existing UI components, intercepting and reacting to browser events, and accessing environment variables. Our filtering algorithm and the test drivers were both implemented as such extensions in Javascript.

\subsection{User Interface Elements}

As a principle, a learning interface agent must remain as unobtrusive as possible, and therefore the user interface additions were kept to a minimum. We have only provided two extra menu items in the browser's context menu:

- one called "Block Me" which appears only when the user right-clicks on an URL (e.g. an image) that he/she wishes to block;

- the other called "Unblock" which is always available should the user want to unblock an URL that appears to be blocked by mistake. Selecting this item brings up the list of blocked items for the page, and the user can then choose which URL needs to be unblocked.

The "Block Me" button is the way the user provides the positive examples to the algorithm, while the "Unblock" button provides the negative ones. One could envisage that the non-blocked items that were correctly classified as such should also be fed to the algorithm (once the user has left the given page, thus confirming that they were correctly left unblocked) for weight reinforcement purposes, but we have decided against it, as we thought that if the user is the sole trigger for example provision, he/she will have a better feel for what is happening behind the scenes. This remark also holds for the blocked items that were not unblocked by the user. Finally, this way the user has control over the creation and potential proliferation of experts, which otherwise could slow down the browser without much benefit. Notice that updates of the expert weights occur only when receiving misclassified examples: false positives decrease the weight of "positive" experts (voting for the "Block" class), while false negatives decrease the weight of negative experts (voting for the "Unblock" class).

\subsection{Implementation of Create_Hypotheses(.)}

To generate the new set of experts $\mathbf{N}$ in Algorithm 1 we tokenize the example URLs using the character " " as delimiter. The tokens obtained represent items such as domain names, folders, but exclude file names. In that last case indeed, filenames are often generated automatically for the URLs to block (e.g. by advertisement sites), and the resulting filenames generally have little significance. Furthermore, this helps to keep the list simple to manipulate manually. This very simple choice of tokenization seems to be chosen by a significant proportion of users sharing their regular expressions on AdBlock's forums. Notice that "http" is also a resulting token. The user may view its weight as the balance between the rate of false positives and the rate of false negatives achieved through learning, or, similarly, as an indication of the ratio between precision and recall. 
The obtained tokens are then compared with the corresponding existing set of experts. By "corresponding" we mean that tokens obtained from positive (resp. negative) examples are compared to the "positive" (resp. negative) set of experts. If no match is found, the new token is added to the corresponding list of experts, and its weight is initialized using Algorithm 2. More tokens could obviously be generated. For instance, we could also use the full URL itself as an expert. Also, the character "." could be used as a delimiter, to help identify the parts of a domain name that are key to its classification (e.g. host name or domain extension). Finally, one could create experts that capture the importance of the order in which significant tokens appear in a URL. The factor to consider however is to avoid the proliferation of experts.

\section{Experimental Results}

In our experiments, we have fixed $\beta=1 / \sqrt{e} \approx 0.61$ in update rule (11). In order to obtain results that are independent from any particular browsing habit, we needed to provide a test setting that could be used seamlessly by any kind of user. To do so, in addition to providing the standalone extension described in the previous section, we embedded our algorithm inside the AdBlock extension code.

The AdBlock user is asked to set up filters as usual in the form of regular expressions, creating as a result an oracle for the embedded learner. The AdBlock filters override the learner's classification in order to remain transparent to the end user. This means that to the user, the extension is behaving no differently than the regular AdBlock. However, all learner misclassifications (i.e. false positives and false negatives) are fed back as such to the algorithm, leading to the expert creation and weight adjustments described above.

At each step consisting of $k$ observations (e.g. visited image URLs), we freeze a copy of the learner's knowledge base up to that point. While the unfrozen version keeps evolving and accepting feedback from the oracle, the frozen copy is used to evaluate the learning accuracy of the accumulated knowledge so far by populating a confusion matrix based on its predictions on the incoming examples. After $n$ such steps, and for a total of $n \times k$ observations, the user is notified that the testing is finished, and the logs are collected. We can therefore compare the learner at each step and observe the evolution of its ability to classify the upcoming observations. However, as we get close the final steps of each test, the number of observations available to the more recent learners decreases, and the statistical confidence in the more recent results decreases as well. To reduce this phenomenon, we allow some more observations to be collected after the last step.

\subsection{AdBlocking on a Single Commercial Website}

Our first set of tests were designed to see whether our algorithm was able to correctly predict which URLs to block on a single "busy" (i.e. littered with annoying images) web page, and if so, after how many visits. We used a common 

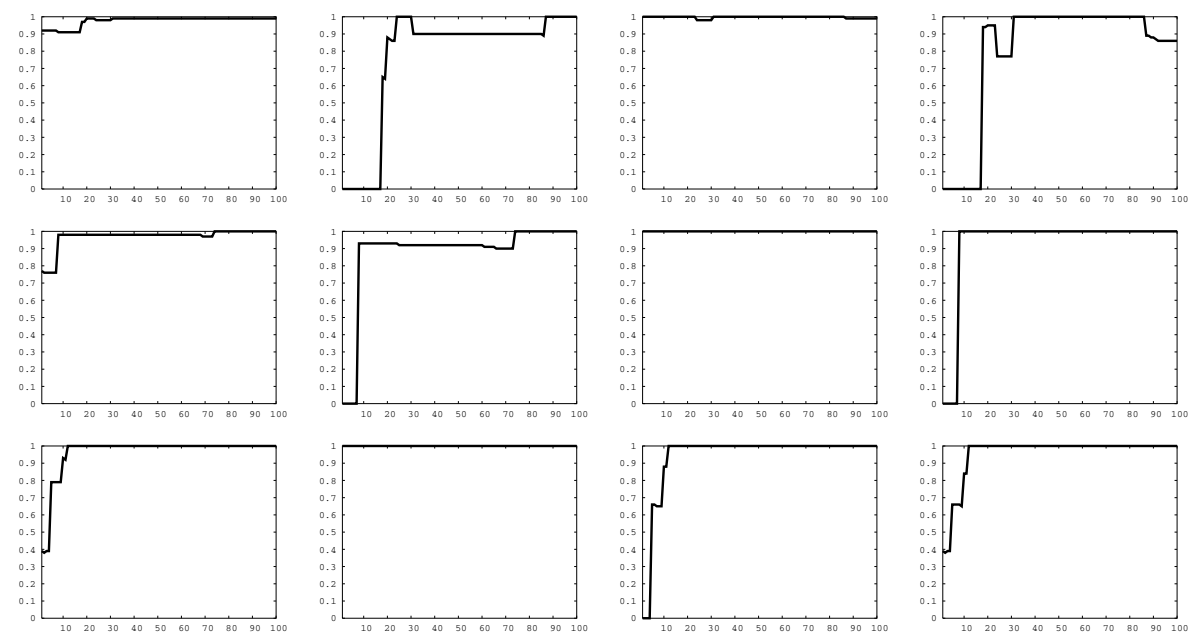

Fig. 2. From left to right: accuracy, recall, specificity and precision. From up to bottom: websites of CNN, Fox News and MSN ( $x$-axis=step number, see text for details).

set of AdBlock regular expressions, as collaboratively devised on AdBlock discussion forums, as oracle. On three popular and large commercial websites, we have run AdBlockLearner with $k=1$ and $n=100$. The total 100 observations were usually reached very quickly. Figure 2 plots the evolution of four key parameters throughout learning. If we denote by $T P$ the number of true positives, $T N$ the number of true negatives, $F P$ the number of false positives and $F N$ the number of false negatives, then the specificity is $T N /(T N+F P)$, the recall is $T P /(T P+F N)$, and the precision is $T P /(T P+F P)$. As can be seen, the algorithm converges quickly to very good prediction, in terms of all four parameters. This is good given that commercial web sites use dynamic loading of advertisements using cookies, and as a result hitting reload usually brings up a different set of images and URLs. However it is important to point out that to obtain similar results in a non-test setting, the misclassifications that were detected by the oracle would have to correspond to as many direct feedbacks by the user. In practice, in the absence of so many interactions, the four parameters can be suboptimal, but it is definitely acceptable.

\subsection{AdBlocking While Surfing to Different Websites}

The next set of tests measures robustness to overfitting. How does the learned knowledge "travel" over to other web sites, are the rules learned so far useful to new websites, and how much more learning is left to do? Our intuition was that the amount of misclassifications would decrease over time, as usually the providers of invasive advertisements are the same in many different commercial sites. We asked the users to simply follow their usual browsing habits, and we 

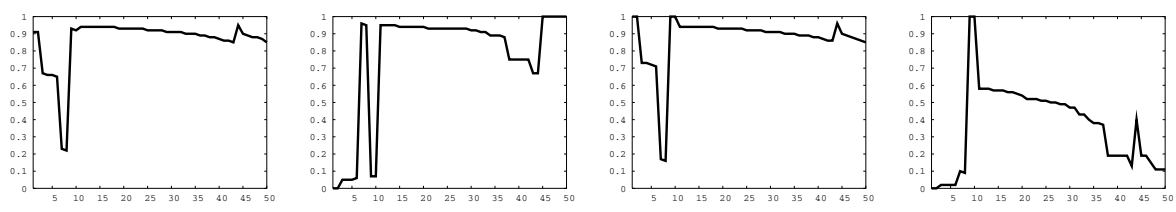

Fig. 3. Evolution of the four parameters during a typical browsing session (conventions follow figure 2 see text for details)

set $k=10$ (to absorb some of the variability) and $n=50$. To make the task harder, we requested that each chosen web site had to be visited only once. The results are charted on figure 3. It is quite remarkable that the accuracy, the specificity and the recall all generally remained at very high values after a short period, given the tough experimental setting. However, the fact that the precision decreases tends to indicate that there is a significant increase in $F P$ (to make the precision decrease) and in $T N$ (to make the specificity remains at high values). This may display the fact that the number of negative examples tends to increase, but the experts might be too simple to fit to the growing amount of information, to discriminate all the examples that come from various websites. In case false positives are deemed unacceptable by the user, i.e. the user does not want to have to manually unblock erroneously blocked URLs, it is possible to alter the utility of the weighted majority vote by giving more weight to negative votes, as also indicated by $[8$. The trade-off would be a drop in overall accuracy and an increase in the rate of false negatives.

\section{Conclusion and Future Work}

In this paper, we have experimentally demonstrated the efficiency of a careful adaptation of weighted majority. Compared to usual weighted majority, our setting makes use of the fact that experts may abstain instead of always predicting a class. This raises an important theoretical issue, as the efficiency of weighted majority is usually measured with respect to its number of mistakes [4]. In our setting, we would certainly appreciate this quantity to be as small as possible, but we would also appreciate the number of abstention to be small. Since mistake bounds do not take into account the number of abstentions, this raises both the problem of finding accurate quantities to minimize, and relevant bounds that our adaptation of weighted majority satisfies.

\section{Acknowledgments and Code Availability}

We would like to thank "Rue", chief developer of AdBlock, for his timely help and enthusiasm, and for welcoming us to his team. R. Nock would like to warmly thank Ottawa University and Stan Matwin for an invitation grant, during which part of this work was achieved. Both the standalone extension 
(AdBlockLearner) and the test driver (AdBlockLearnerTest) are available at http://adblocklearner.mozdev.org, including source code and documentation. They are compatible with most versions of Mozilla Firefox.

\section{References}

1. AdBlock, 2005. http://adblock.mozdev.org.

2. Jeffrey E. F. Friedl. Mastering Regular Expressions. O' Reilly, 1997.

3. Y. Lashkari, M. Metral, and P. Maes. Collaborative interface agents. In Proc. of AAAI-94, pages 444-449, 1994.

4. N. Littlestone and M. Warmuth. The weighted majority algorithm. Information and Computation, pages 212-261, 1994.

5. Mozilla Firefox, 2005. http://mozilla.org/products/firefox.

6. Mozilla Firefox Extensions, 2005. http://update.mozilla.org/extensions/.

7. R. Nock and B. Esfandiari. Oracles and assistants : machine learning applied to network supervision. In Canadian Artificial Intelligence Conference, number 1418 in Lecture Notes in Computer Science, pages 86-98. Springer-Verlag, 1998.

8. M. Sahami, S. Dumais, D. Heckerman, and E. Horvitz. A bayesian approach to filtering junk email. In AAAI Workshop on Learning for Text Categorization. AAAI Press, 1998. 